\title{
Nutrition and immunity
}

\section{By D. ParratT, Department of Bacteriology, Ninewells Hospital, Dundee DDI $9 S Y$}

This paper considers some aspects of antimicrobial immunity which are, or may be, modified by nutritional deficiencies. The starting point has to be an acceptance that all humans are challenged by resident micro-organisms every minute of their lives. It is clear for example that at the moment of death, when the checks have been removed from these micro-organisms, there is decomposition of the body by the multiplying organisms. The organisms in this instance come from internal and external body surfaces, (skin, gut, mouth etc.) and are normally referred to as commensal micro-organisms, i.e. permanent residents of our body, which do us no harm. However, they fail to do us harm because they are, in life, carefully counterbalanced, and the immunological system is one part of this balance. A failure in the immune response will, therefore, often be reflected by infection due to these commensal micro-organisms, which is often termed an 'opportunistic infection' or 'infection by an opportunist'. There is no justification for the use of these terms, and they should be removed from our scientific vocabulary as soon as possible. At best the use of such terms, provides only meaningless description, whilst at worst it detracts our attention from the balance between host and organisms, breakdown of that balance, and any attempts at understanding the processes which maintain the balance. It must be recognized, though, that the term 'opportunistic infection' was primarily introduced to differentiate infections caused by commensals from the more classical infections, such as typhoid, smallpox, measles, scarlet fever, etc., where high infectivity, often with considerable mortality, and a specific pattern of onset and course of illness, were seen. In many of these 'classical' infections, a specific mechanism of damage (e.g. the action of a toxin, or invasive potential) was demonstrable. It has become a habit in microbiological investigation to search for such mechanisms wherever an organism is producing disease and the process has been applied even for the 'opportunists' themselves. Not surprisingly, 'toxins', here used in the widest sense to mean a product of an organism which damages, paralyses or interrupts some physiological function of the host, have been demonstrated for most bacteria, fungi and some protozoa, and disease has been ascribed to them. All of this has obscured the most important fact, referred to above, of balance between host and micro-organism. Failure to check a microorganism's growth will have similar consequences whether it is a classical pathogen or a commensal. It is not toxin, or any other 'virulence factor' of the classical pathogen which gives it a lethal capacity, but rather its ability to overbalance the checks which the host may produce against it. The classical pathogens have one advantage over the commensals in this - unfamiliarity. The 
host has never had an opportunity to learn how to counter-balance these organisms, as it has in the case of commensals. Indeed, as we know, it is possible for most 'pathogens' to be reduced to the status of commensals. Examples are typhoid bacilli, pneumococci, streptococci, tubercle bacilli, measles virus etc. Likewise, by changing the host-parasite balance, something which the modern clinician finds unavoidable, commensal micro-organisms become 'pathogens'. An example here would be fungal infection after immunosuppressive therapy. The important point is that the organisms themselves may not be changed, and sometimes the host is not changed but the balance between them is changed and this determines the outcome for the patient.

It follows, therefore, that to understand infections we must look at the nature of this balance-how it is established and maintained, or how it is lost and the consequences thereof. One way to do this is to consider the position illustrated in Fig. I.

Immunity in the context of this discussion implies control (i.e. balance) of the population level of the micro-organisms, without damage to any host tissues or processes. It is important to note that 'immunity' does not mean eradication of the organism - only control of its population, and this can be control of a large or small population. Indeed most commensals, in health, are controlled at large population densities. A response which is less than this, must by definition, imply a lack of complete control, and this is an immunodeficiency. Many, but not all infections, are due to such a lack of control and it is this area, between deficiency and immunity which will be discussed in the remainder of this paper. Nevertheless, it is important to realize that in the case of almost all infections (whether by 'pathogens' or commensals) the host response begins in the 'deficiency' category and changes with time into an 'immunity' response. It is conversely true that responses which begin with 'immunity' status may revert to a deficiency status.

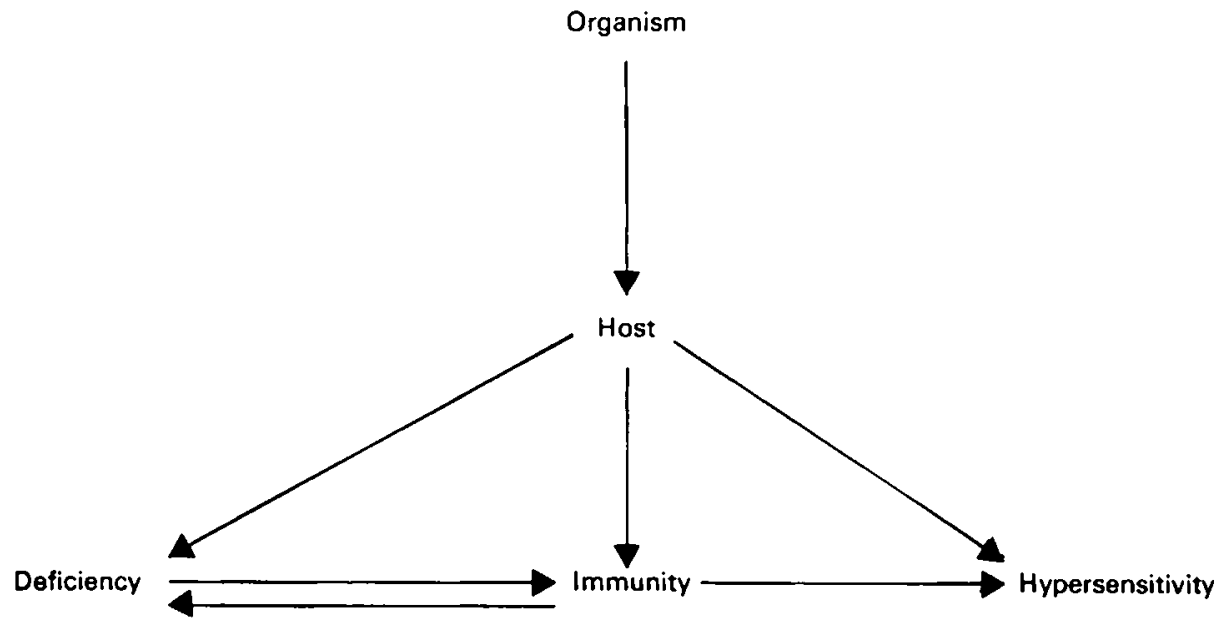

Fig. I. Schematic diagram to illustrate the possible interactions between a micro-organism and its host. 
The third category in Fig. I, that of hypersensitivity, is of importance because we are at least beginning to recognize that the damage to the host in an infection is often due to hypersensitivity. We can define hypersensitivity in the present context as a state where the population size of the organism may or may not be controlled, but where there is always self-inflicted host damage resulting from the response to the organism. In infection, such a state can be seen as 'the last throw of the dice' by an immune response which has failed to gain control by ordinary means and has upgraded its activity even at the risk of self-damage. It is important to recognize that damage due to this sort of process can provide a rational basis for understanding why organisms devoid of toxins, enzymes or other aggressins are perfectly capable of producing severe, and sometimes fatal, disease. The fungi, particularly Candida albicans and Aspergillus species, are notable in this context. Interesting though this topic may be, it is not the one with which this article is concerned and we must return to a consideration of the differences between immunity and immune deficiency.

\section{Immunity}

Let us consider immunity first. In the case of $C$. albicans, a yeast, which is constantly represented in the oral and gut flora of normal individuals, there is a clear balance maintained over long periods. In Fig. 2 the main features of this balance can be seen. The antibody levels here have been measured very accurately in one individual by a radioimmunoassay technique and it is clear that antibody varies very little in amount over long periods of time. Similarly, salivary colony counts of the organism ( $C$. albicans), which presumably reflect the counts in the rest of the intestinal tract and perhaps elsewhere, do not vary greatly. This is the pattern which is seen in all normal individuals, although the actual counts of C. albicans and the corresponding antibody levels vary from individual to individual. However, this balance between organism and host is not a static one. The organism constantly attempts to increase its population size and the immune system constantly responds to this. This response and anti-response is reflected in Fig. 3, which shows the rate of change of antibody level in a typical normal host response. Upward deflection represents an acceleration of response, downward deflections-deceleration of the response. It is clear that whilst there is a constancy of response, this is achieved by reciprocal accelerations and decelerations. It is this precarious and finely-tuned balance which must be maintained if the host is not to be overwhelmed. The discussion above has considered only antibody responsiveness but it is reasonable to suppose that if antibody is responding in such a way, other parts of the immune response will be responding similarly. Thus, phagocytic function, and T-cell (cell mediated) functions will be similarly balanced. Indeed, because true responsiveness on the part of phagocytes (polymorphonuclear leucocytes and macrophages) is essentially a function of antibody and T-cell activity respectively, this is not too severe a restriction on the original assumption. 
No.

organisms/ml Antibody $(\mu g / m l)$

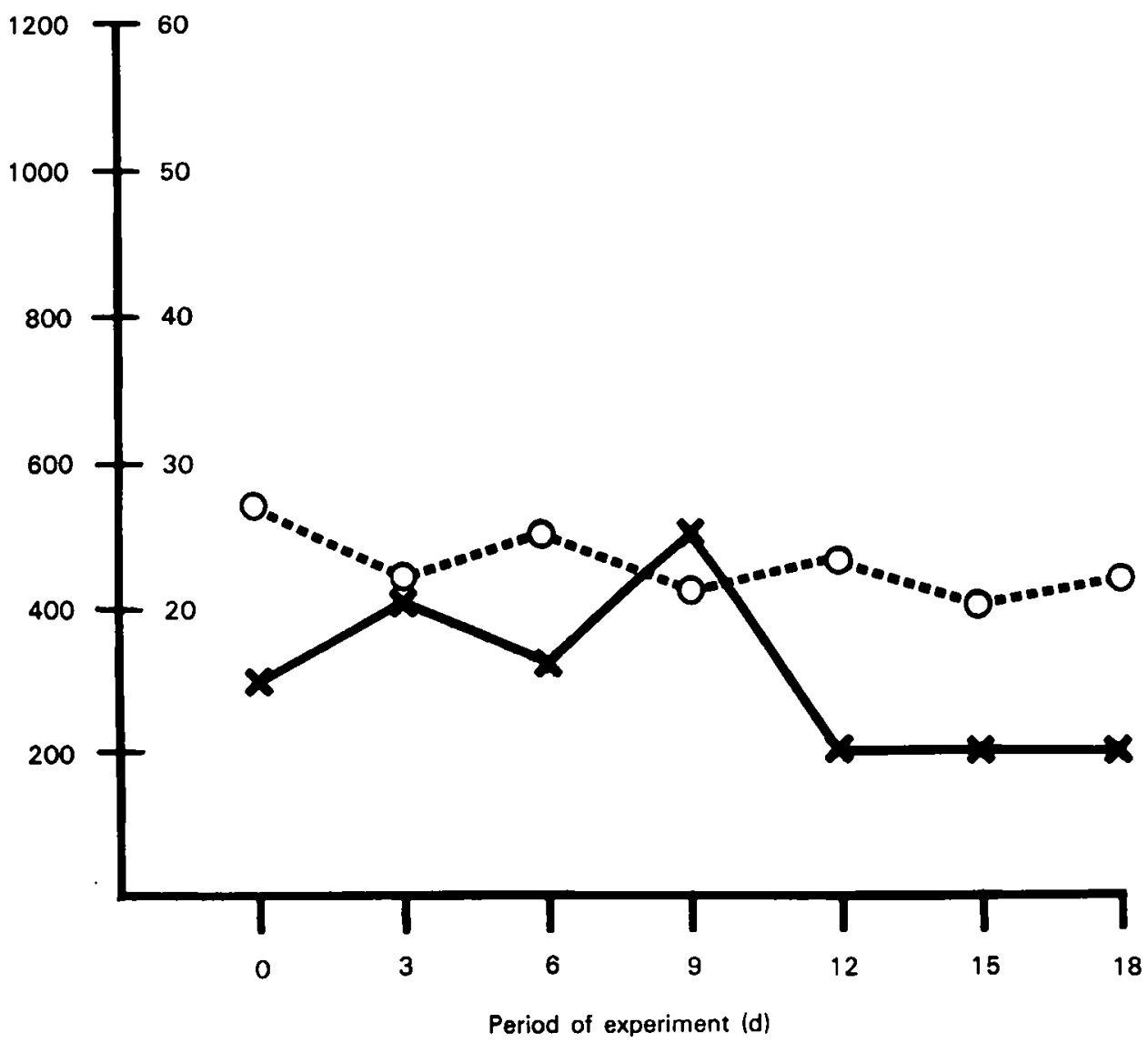

Fig. 2. Anti-Candida antibody $(O---O)$ and salivary Candida albicans colony counts $(x-x)$ in a human volunteer during a serial estimation.

\section{Nutritional deficiencies}

Many manipulations of the immune response such as immunosuppressive therapy, antibiotic therapy, blood or blood-product transfusion, and plasmapheresis, may alter such a balance and in respect of Candida infection it is a common experience that some of these manipulations result in infection. What we are concerned with here, though, is what is likely to occur if nutritional manipulations/deficiencies occur (see Dick (I979) for useful reviews).

Bearing the balance in mind, and remembering that balance has to be achieved for incoming pathogens, what is the effect of depletion of fuel for the immune response? Severe protein-energy malnutrition, of the type seen in underdeveloped countries is notable for its effect on specific responsiveness, i.e. on the balancing between organism and host. For example, the action of polymorphonuclear leucocytes is essentially normal (a non-specific or at least non-responsive action). 


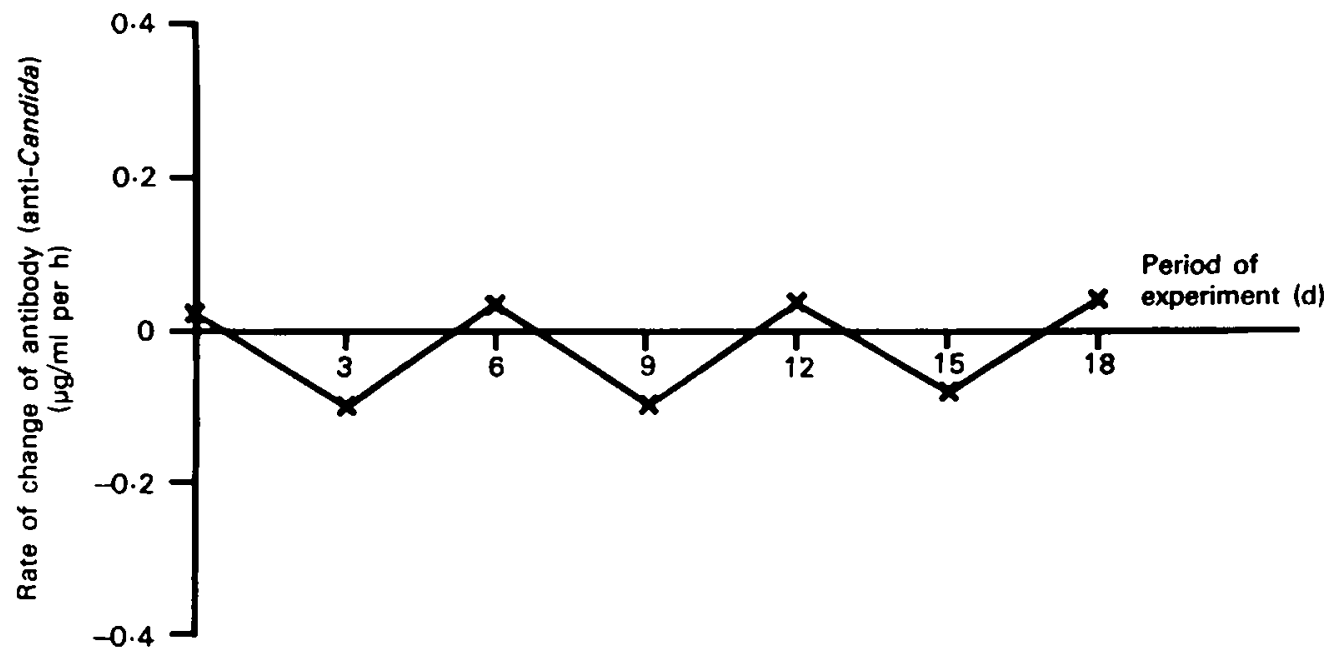

Fig. 3. Changes in anti-Candida antibody synthesis $v$. time. (Values are for the same subject and period as in Fig. 2).

Similarly, total immunoglobulin levels (which do not reflect specific immunity) are high rather than low and yet the formation of antibody (to vaccine) has been shown to be impaired. T-cell immunity, which provides both active anti-microbial immunity and control of the humoral and cell-mediated response, is seriously deficient in protein-energy malnutrition. Clearly any hope of achieving balance is going to be remote in these patients and the loss of control can result, predictably, in a predisposition to frequent and serious infections. Here we see perhaps the difference between immune parameters in the form of total function and responsive function in a more specific sense, the difference between responding and responding well. This is the extreme, to be borne in mind perhaps in seriously ill patients in this country who require full supportive intravenous feeding. However, there are lesser degrees of nutritional deficiency which may have significant effects, and notable amongst these are mineral and vitamin deficiencies.

Zinc deficiency is perhaps the best defined from the point of view of immune deficiency. It is well established that nutritional deprivation of zinc in mice leads within $28 \mathrm{~d}$ (Frater et al. 1977) to severe deficiencies of cell-mediated (T-cell) immunity. In man it is also clear that $\mathbf{Z n}$ deficiency leads to T-cell failure (Golden et al. 1978) and probably to a predisposition to infection. In both the animal and human studies so far reported the effect of $\mathrm{Zn}$ deficiency has been severe, but we have to ask whether a minor degree of deficiency, whilst not producing severe failures of T-cell function, may produce sufficient disturbance to lead to an imbalance between host and micro-organism. This is particularly important when one considers the delicate balance between the host and commensal microorganisms, and the fact that it may be difficult to maintain normal $\mathrm{Zn}$ homoeostasis in seriously ill patients.

With another mineral, iron, deficiency can precipitate infection with otherwise harmless organisms such as $C$. albicans. It is known for example that at least a 
third of patients with recurrent oral candidosis can be effectively treated with Fe. Whether the deficiency of $\mathrm{Fe}$ in this case alters immune responsiveness, or simply repairs deficiences in the oral mucosa is uncertain, but the important point is that the balance between host and organism is restored.

The position of Fe deficiency is not, however, as simple as this example may suggest. It is well established that micro-organisms require iron for their own metabolism and that they possess special protein siderophores for obtaining this $\mathrm{Fe}$. Without $\mathrm{Fe}$, they cannot survive, and it has been postulated that $\mathrm{Fe}$ deficiency may therefore protect the patient from infection, whilst diseases associated with high body $\mathrm{Fe}$ loads may predispose to infection. There is evidence to support both of these possibilities in man but it must be noted that the information is crude, relying only on a difference in incidence of infection between either Fe-deficient or Fe-loaded patients and normals. Such assessments are crude compared with the measurement of the balance between an organism and its host of the type illustrated earlier. It seems more likely that changes in immune responses would be clearer if the balance was studied in patients with disturbances of iron metabolism. More investigation would be desirable in this area, but it must be accepted first that the relationship between an organism and its host is a delicate one, and cannot be assessed by crude morbidity or mortality information. $A$ refined approach to the microbiology and immunology is necessary.

Similarly, a deficiency of folic acid may lead to chronic candidosis. The possibilities regarding the effect on immune response or mucous membranes or both are the same as for $\mathrm{Fe}$, as are the implications in respect of disease or health.

There is also the vexed question of vitamin $\mathrm{C}$ and its effect on immunity. Initial reports concentrated on the response to the common cold, in individuals given a diet high in vitamin C. The result of several such reports seems to indicate that whilst the vitamin does not prevent infection it certainly modifies the infection by reducing the symptoms and the period of infection. Further, it is of interest that a defined role for vitamin $\mathrm{C}$ has now been shown in its action on polymorphonuclear leucocytes (Sandler et al. 1975). The action and importance of vitamin C in other infections has not been thoroughly researched and it may be that minor immune deficiencies due to an inadequate supply of the vitamin, might sufficiently interfere with the balance of host parasite interplay to cause infections by commensal microorganisms.

To return to the problem of protein-energy disturbance, it is well established for example in diabetes, nephrotic syndrome, and malabsorption syndromes, where such disturbances are common, that infection is a serious and chronic problem. Unfortunately the reasons for such increased susceptibility are not obvious. On the one hand the deficiency could arise because of the metabolic disturbance, on the other it is possible that the pathological processes in these diseases also produce a defect in the immune response. Indeed if we pursue this line of thought it is a good generalization that all individuals with severe and chronic diseases (of a non-infective nature) are more than usually susceptible to infection. It is further true that individuals infected with one 
organism may become susceptible to secondary infection with another. For example, influenza predisposes to infections such as those due to herpes simplex virus. The picture in all of these instances is complex but we can reasonably assume that the disease processes are lowering immune responsiveness to the point where an effective challenge towards another organism cannot be balanced by the immune response. Work by the author's group (unpublished results) has concentrated on attempting to define the degree of efficiency which is required to balance, and therefore control the anti-microbial response. This has been done by using results obtained from the study of commensal micro-organisms such as C. albicans, and the responses made to them when an induced population increase of the organism occurs. The results are projected with the aid of computer analysis. The information is primitive at the moment but several interesting aspects have come to light. For example, an immune response has to be about $97 \%$ efficient/unit time in its killing of the organism (unit time here refers to the division period of the organism). A response less effective than this cannot 'catch' the expanding population of micro-organisms before overwhelming (lethal) populations of the organism are reached. Indeed, as will be obvious, in order to balance the population increase, a response of $100 \%$ efficiency is required (i.e. all the new organisms produced in one unit of division time are destroyed) and a response greater than $100 \%$ is needed to eliminate the organism. The problem for the immune response is in accelerating sufficiently to change its efficiency from about 97 to $100 \%$ or more. This may sound simple, but in practice it takes on average $7-14 \mathrm{~d}$ to achieve and the control of the final 'thrust' is delicately organized. It depends on optimum tuning of the response and therefore can be deranged by many abnormalities of physiological function. Nutrition is important here because an immune response which is not supplied with the necessary building blocks, energy, or catalysts will clearly be incapable of its maximum efficiency. The provision of normal nutritional status is therefore of paramount importance in all diseases, if infection is to be avoided This article has not attempted to review all of the associations between nutritional deficiency and infection because these factual reports do not help to understand the processes which result in infection. Rather, an attempt has been made to indicate the direction to be followed in the investigation of immune responsiveness and particularly in its relationship to nutritional deficiency. The complexities of nutritional disturbances cannot for reasons of space be considered here but it is reasonable to conclude that a coordinated effort by nutritionists, immunologists and microbiologists is required if the problems are to be solved.

The position of the microbiologist, though, must be more active than it has been in the past. Documentation of infections in different diseases, and classification as 'opportunistic' or 'pathogenic' organisms has not provided a useful approach to patient management. A new approach based on the determination of the efficiency or inefficiency of host response must be found, and using this an effort must be made to correct any defects of the response. This will not be easy, for in philosophical terms it is a new avenue never before followed by microbiology and 
one which departs from the classical doctrine of isolation, identification and sensitivity testing'. The new approach has to answer the question; is the population size of this organism controlled or not? If it is not, control must be supplied, and somewhere along this difficult course it will be necessary to decide whether nutritional status, in its widest sense, is the cause of impaired efficiency of the response.

\section{REFERENCES}

Dick, D. (Editor) (1979). Immunological Aspects of Infectious Disease. Lancaster: M.T.P. Press. Frater, P. J., Hass, S. M. \& Luecke, R. W. (1977). \%. Nutr. 107, 1889.

Golden, M. H. N., Golden, B. E., Harland, P. S. E. G. \& Jackson, A. A. (1978). Lancet i, 1226. Sandler, J. A., Gallin, J. I. \& Vaughn, M. (1975). F. Cell. Biol. 67, $4^{80 .}$ 